\title{
Una revisión sobre los programas alimentarios nacionales aplicados a comedores escolares y comunitarios desde los años ochenta en Argentina*
}

\author{
María Victoria Sordini*
}

\section{Resumen}

El presente trabajo tiene por objetivo reflexionar en torno a las políticas alimentarias nacionales aplicadas en comedores escolares y comunitarios desde los años ochenta.

Se describió la problemática alimentaria, la trayectoria de los comedores escolares, el surgimiento de los comedores comunitarios y las políticas aplicadas a su financiamiento.

La metodología utilizada fue la revisión bibliográfica sobre las políticas alimentarias de alcance nacional en Argentina desde 1983. La lectura cronológica permitió identificar continuidades y rupturas en la gestión de los programas.

Entre los resultados se destacó que los programas alimentarios fueron formulados para contener situaciones de emergencia, sin embargo, los comedores continúan brindando asistencia alimentaria. También se reflexionó en torno a la malnutrición y a las prestaciones alimentarias precarias que ofrecen los comedores, repitiendo las pautas de las comidas "rendidoras", que utilizan como estrategia de consumo los hogares de bajos ingresos.

\footnotetext{
* Artículo recibido el 30 de junio de 2014. Aceptado el 21 de agosto de 2014.

* María Victoria Sordini es estudiante avanzada de la carrera Licenciatura en Sociología de la Universidad Nacional de Mar del Plata.

Correo electrónico: mvsordini@hotmail.com.ar
} 


\section{Palabras clave}

Problemática alimentaria - comedores escolares y comunitarios - programas alimentarios.

\section{Abstract}

This essay aims to reflect on national food policies in school refectories and community kitchens since the eighties.

Food problems, the trajectory of school refectories, the emergence of community kitchens and their financing policies are described.

The methodology used was the bibliographical revision on food policies at national level in Argentina since 1983. Chronological reading allowed identify continuities and ruptures in the management of programs.

Among the results it should be noted that food programs were formulated to restrain emergency situations, however, community kitchens continue providing food assistance. Was also considered poor nutrition and precarious food benefits that community kitchens offers, repeating guidelines of carbohydrate and fats foods meals, using as strategy of consumption by low-income households.

\section{Keywords}

Food problems - School refectory and community kitchens Food programs

\section{Introducción}

Con la obligación de hacer cumplir el derecho de todos los habitantes a una alimentación suficiente, nutricional y culturalmente adecuada, el Estado ha implementado diversas políticas sociales y programas alimentarios, entre ellos, el financiamiento a comedores escolares y comunitarios.

El presente trabajo tiene por objetivo reflexionar en torno a las políticas alimentarias nacionales aplicadas a los comedores escolares y comunitarios desde los años ochenta.

En un primer apartado se describirá la problemática de la malnutrición como un problema social.

En segundo lugar se hará un breve repaso por la historia de los comedores escolares. $\mathrm{Y}$, a continuación, se describirá el surgimiento de los comedores 
comunitarios como una estrategia de organización colectiva ante las necesidades alimentarias.

En un tercer apartado se describen los programas alimentarios vinculados al financiamiento de comedores escolares y comunitarios de alcance nacional en Argentina desde el retorno a un gobierno democrático.

Finalmente se reflexionará acerca de las trayectorias de los programas nacionales, su vinculación con la problemática alimentaria y la consolidación institucional que adquirieron los comedores comunitarios.

Este trabajo constituye un avance de la tesina de grado, que apunta a reflexionar y establecer conclusiones preliminares sobre los programas alimentarios nacionales desde los años ochenta.

\section{La problemática alimentaria}

Entre las desigualdades sociales generadas por el capitalismo, la malnutrición refleja una clara dificultad de acceso a los recursos, siendo que amplios sectores de la población, como afirma Patricia Aguirre (2005), no comen lo que quieren ni lo que les hace bien, sino lo que pueden.

En los sectores de bajos ingresos la alimentación se configura en torno a 'lo posible', esto tiende a ser alimentos ricos en carbohidratos -complejos y simples- y en grasas, pobres en fibras, proteínas de alto valor biológico y micronutrientes. La persistencia en el tiempo de este tipo de alimentación, más allá de clausurar oportunidades de comer diferente, trae consigo sobrepeso, obesidad y desnutrición oculta; es decir, las otras posibles formas que adquiere la malnutrición, más allá de la desnutrición aguda (Scribano, Eynard y Huergo, 2010).

La mala alimentación repercute en la formación de los cuerpos, condicionando la estatura, la dentadura y el desarrollo cognitivo. Esta debilidad en los cuerpos genera debilidad en las interacciones sociales que constituyen, sostienen y reproducen a la sociedad.

Como sostiene Aguirre, la malnutrición es una problemática social que tiene su origen en la modificación de las relaciones sociales que han sufrido las sociedades bajo el imperio del mercado globalizado, que afecta principalmente a los pobres y que continúa creciendo porque es funcional al sistema social actual. (Aguirre, 2011)

\section{Breve revisión de los comedores escolares desde inicios de siglo XX}

La ayuda alimentaria en Argentina tiene sus raíces en las escuelas. Según se constata en los trabajos del Centro de Estudios sobre Nutrición Infantil (CESNI), alrededor del año 1900 se detectó un elevado porcentaje de ausentismo, sumado a un escaso rendimiento intelectual debido a que los niños concurrían a la escuela 
insuficientemente alimentados. En 1906 se instala el primer servicio de "Copa de leche" en Buenos Aires y dos años más tarde se instala en Rosario. En 1914 se incluye por primera vez en los presupuestos del Consejo Nacional de Educación una partida anual para alimentación en las escuelas de la Capital Federal y del territorio nacional. En 1932 se sanciona la ley 11.597, mediante la cual se proporcionan fondos para el mantenimiento de los comedores escolares dependientes del Consejo Nacional de Educación y del Instituto Nacional de Nutrición. En 1938 se creó la Comisión de Ayuda al Escolar, que proveía ropa, alimentos y medicamentos. A su vez, se habilitaban comedores escolares en todo el país. En 1943 por decreto nacional la Comisión Nacional de Ayuda Escolar pasa a ser una institución integrante de la dirección Nacional de Salud Pública y Asistencia Social. En 1945 pasa a depender de la Dirección General de Asistencia Social y a llamarse Dirección de Ayuda Escolar. En 1964 se promulga la implementación de la copa de leche en todas las escuelas dependientes de la Dirección General de Escuelas.

El hito trazador de la historia reciente de los comedores escolares en Argentina probablemente sea el Programa de Ayuda a los ingenios azucareros de Tucumán, en 1967, que instituye su funcionamiento, primero en esa provincia para extenderse luego y en forma progresiva al resto, alcanzando a todo el país recién en 1984. (Britos, O'Donnell, Ugalde y Clacheo, 2003: 15) con el nombre de Programa de Promoción Social Nutricional (PROSONU)

\section{El surgimiento de los comedores comunitarios: la organización colectiva de las necesidades}

En 1989 y 1990 Argentina transitó momentos de alto índice de desempleo, brusco aumento de precios (hiperinflación) y muy bajos salarios. La hiperinflación representa el

... momento en que técnicamente las mercancías no tienen precio y una moneda determinada ha dejado de funcionar como equivalente general: socialmente, el momento en que se ha roto lo que aparece mediando todas las relaciones que forman la base de la sociedad capitalista. (Iñigo Carrera, Cotarelo, Gómez, Kindgard, 1995: 62)

Es decir, la hiperinflación significa que la mediación de todas las relaciones sociales en la sociedad capitalista, esto es, el dinero, ha dejado de existir.

Ante el deterioro de los ingresos de importantes segmentos de la sociedad, surgieron -en algunas áreas geográficas con alta presencia de sectores pobresacciones precarias y espontáneas, autogeneradas por los vecinos $y$, en algunos 
casos, con la participación de ONG. Bajo el nombre de 'ollas populares' ${ }^{1}$ o 'comedores comunitarios', estas acciones estaban destinadas a contener los apremios alimentarios más urgentes, pero tenían una cobertura y una capacidad de ofrecer alimentos muy limitadas. (Vinocur y Halperin, 2004: 28)

Como afirma Massetti (2011),

... frente a la caída del ingreso o a la inestabilidad laboral primero y luego frente al hiperdesempleo, el "barrio" comienza a convertirse en un componente fundamental en las estrategias familiares de supervivencia de los sectores populares. Tanto sea a nivel simbólico como material. Comienza a percibirse un fenómeno que podemos llamar el "ingreso barrial"; esto es, estrategias comunitarias de satisfacción de necesidades. Qué comienza a generalizarse hacia finales de los 80 con la hiperinflación: ollas populares primero, y luego comedores comunitarios, roperos, compras comunitarias, huertas comunitarias, etc. (Massetti, 2011: 11)

Los sectores populares, resistiendo a las políticas neoliberales, se organizaron de modo colectivo para dar respuesta a las necesidades más urgentes. Si bien estas formas de asociación surgieron como prácticas temporarias, se consolidaron durante la década del noventa como consecuencia de la continuidad de los procesos de pauperización y de los programas de apoyo a comedores comunitarios implementados.

Hacía principios del milenio emergieron nuevas organizaciones colectivas en torno a las necesidades más urgentes debido a la crisis. Hacia finales del año 2001 el índice de pobreza alcanzaba el 38,3\% de la población urbana, incrementándose aún más hacia 2003 con un $54,7 \%{ }^{2}$ Este período también fue acompañado por planes nacionales que promovieron el desarrollo de los comedores comunitarios acompañados por ONG.

\section{Políticas alimentarias nacionales aplicadas a comedores escolares y comunitarios desde los años ochenta}

Desde los años ochenta, en el marco de políticas privatizadoras, se instalaron las tendencias a focalizar las políticas sociales en las poblaciones más vulnerables, mediante programas selectivos, y desmantelando los programas universales. "Se configuró el ámbito de acción de la política social como subsidiario en materia de pobreza, consolidando el reemplazo de la concepción de la universalidad por el de focalización" (Sojo, 2007 citado en De Sena, Cena, 2014: 22).

\footnotetext{
${ }^{1}$ Las ollas populares constituyen una de las formas más emblemáticas de la ayuda alimentaria. Sus orígenes en Argentina remontan a la década del 30, como consecuencia de los sucesos comprendidos en la depresión económica mundial. Décadas después las ollas populares acompañaron el proceso de marginalidad social en los cinturones periurbanos de las grandes ciudades. (Britos et al.2003: 24)

${ }^{2}$ INDEC. Porcentaje de hogares y personas bajo las líneas de pobreza e indigencia en los aglomerados urbanos y regiones estadísticas, desde mayo de 2001 en adelante.
} 
El Programa de Comedores Escolares, desde inicios del siglo XX, partió de una concepción universalista de la cobertura, pero se modificó en los últimos treinta años para privilegiar a las escuelas o a los centros de salud de las áreas consideradas "con desventajas sociales".

El Programa de Comedores Escolares se generalizó a todo el país en 1984 con el nombre de Programa de Promoción Social Nutricional (PROSONU). Se instaló con el objetivo de disminuir las condiciones deficitarias de la alimentación de los niños en edad escolar, con la intención de contribuir a un mayor rendimiento y a la disminución del ausentismo.

En los años noventa

... el modelo de organización económica y social que se impuso en el país centrado en la transferencia de poder y recursos al sector privado, también impulsaba la transferencia de poder y recursos al interior del aparato estatal, disminuyendo la relevancia del gobierno central y creciendo la de los gobiernos provinciales y municipales. (Vinocur y Halperin, 2004: 53)

En este contexto, en 1992 el PROSONU fue descentralizado y el Gobierno Nacional perdió la capacidad de programación, supervisión y evaluación del funcionamiento del programa en cada jurisdicción. En 2002 se estableció la libre disponibilidad de los fondos coparticipables, con afectación específica hasta ese momento, lo cual le otorga flexibilidad a cada provincia para utilizar esos fondos con otros propósitos. Para compensar esa flexibilidad se creó el Programa de Mejoramiento a los Servicios Alimentarios $^{3}$ en las escuelas, dependiente del Ministerio de Educación. (Britos et al. 2003: 18)

Durante la década del 90 dos programas del Gobierno Nacional incidieron en el desenvolvimiento de comedores infantiles y comunitarios: el Programa Materno Infantil y Nutricional (en adelante PROMIN) y el Programa Alimentario Nutricional Infantil (en adelante PRANI).

En 1993 se implementó el PROMIN como un programa focalizado en áreas de alta incidencia de pobreza urbana, que cuenta con el financiamiento parcial del Banco Mundial y tiene el propósito de reforzar las acciones regulares del Programa Materno Infantil, ${ }^{4}$ mejorando la infraestructura y equipamiento de los efectores del primer nivel de atención de salud, articulando una red con Centros de Desarrollo Infantil (CDI) y modificando el modelo de atención y la gestión de los recursos. (Britos et al. 2003: 30)

\footnotetext{
${ }^{3}$ Constituye fondos presupuestarios nacionales, transferidos a las provincias en calidad de refuerzo económico para el sostenimiento de las prestaciones alimentarias en más de 11000 escuelas que atienden a la población de bajo nivel socioeconómico. (Britos et al. 2003: 18)

${ }^{4}$ Su eje central es la distribución de leche a mujeres embarazadas y niños hasta los dos o los seis años de edad. Estos últimos en caso de diagnóstico antropométrico de desnutrición. Desde los años treinta aún está en vigencia.
} 
En primer término una de las líneas programáticas del PROMIN contempló el fortalecimiento de comedores infantiles y su transformación en CDI, incorporando pautas de funcionamiento de la prestación alimentaria y capacitación a madres voluntarias para la organización del servicio alimentario, además de un ámbito institucional y un modelo de atención del desarrollo psicosocial de niños de entre dos y cinco años.

Entre 1993 y 2000, incluyó entre sus acciones la identificación de mujeres embarazadas y niños con desnutrición y su rehabilitación nutricional, financiando la adquisición, en las provincias contempladas, de un módulo de alimentos o caja familiar de alimentos secos. Este complemento se sumaba a la leche que regularmente adquirían las provincias con las transferencias del Ministerio de Salud. Sin embargo,

...una evaluación interna del PROMIN realizada en 1999 no halló resultados satisfactorios, cambios en el perfil nutricional de los niños atribuibles al reparto de cajas, por lo que desde principios de 2000 PROMIN discontinuó esa actividad y se sumó a partir de 2001 a la entrega de leche fortificada. (Britos et al. 2003: 31)

En 1996 se implementó el PRANI, que, en términos similares al PROMIN, se proponía adecuar y transformar el modelo de atención de los comedores infantiles hasta convertirlos en Centros de Cuidado Infantil (CCI). Sin embargo, "ninguno tuvo éxito en dejar establecido un modelo de organización y niveles adecuados de cobertura alimentaria" (Britos et al. 2003: 29).

En el PRANI se establecieron tres líneas de acción: 1) Alimentación y nutrición, 2) Desarrollo infantil y 3) Movilización y participación social. Sus destinatarios fueron niños de entre dos y catorce años, pertenecientes a hogares con necesidades básicas insatisfechas (NBI), residentes en áreas urbanas y rurales con un mínimo de $30 \%$ de población con NBI en relación con el total del área. El programa propendió a la auto focalización, ya que existía una asistencia espontánea de los niños de esa edad a los comedores infantiles. (Vinocur y Halperín, 2004: 29)

Como afirman estos autores, la prestación consistió en el envío periódico de alimentos a las provincias. Por su parte, los comedores escolares e infantiles recibieron financiamiento de los gobiernos provinciales, municipales $y$, en algunos casos, de las ONG. La caja de alimentos, contenía 9 alimentos no perecederos equivalentes a 1.250 calorías, correspondientes a un cálculo nutricional destinado a satisfacer el $77 \%$ de las necesidades diarias de la población destinataria.

En el nuevo milenio, desde 2002 y hasta la actualidad se desarrolla el Fondo Participativo de Inversión Social (FOPAR). Es el tercer programa ${ }^{5}$ que incidió en el desarrollo de los comedores infantiles y comunitarios. En el marco del Programa de

\footnotetext{
${ }^{5}$ Después del PROMIN y el PRANI en los años noventa.
} 
Emergencia Alimentaria ${ }^{6}$ brinda asistencia técnica y financiera a comedores comunitarios y organizaciones de la sociedad civil para la mejora y ampliación de servicios alimentarios, reaparición y/o adecuación de la infraestructura existente y equipamiento básico, con el financiamiento del Banco Mundial con contraparte de aporte nacional. $^{7}$

Las ONG deben presentar Proyectos de Prestaciones Alimentarias Comunitarias, los cuales son evaluados por el FOPAR, que luego transfiere recursos financieros y efectúa el control. La ONG solicitante administra los recursos del proyecto y presenta las rendiciones correspondientes, asegura la calidad y cobertura de las prestaciones de los comedores, y ejecuta actividades de apoyo y desarrollo organizacional de los comedores del proyecto. (Vinocur y Halperín, 2004: 32)

\section{Consideraciones finales}

En primer lugar es pertinente considerar que gran parte de los comedores comunitarios que emergieron durante los años noventa y en el marco de la crisis de 2001 continúan brindando asistencia alimentaria en los barrios carenciados, así como también lo hacen los comedores escolares.

Los comedores comunitarios, "salvo algunas excepciones, no surgen como una ampliación de las actividades de una institución consolidada, sino que generan una institucionalidad propia asociada a la emergencia y con una expectativa de transitoriedad que luego se resigna" (Clemente, 2010: 163 citado por Ierullo, 2010).

Reflexionar sobre el diseño de las políticas alimentarias aplicadas a los comedores escolares y comunitarios desde 1983, por un lado permite registrar una permanente presencia de programas, que, en tanto políticas focalizadas, han sido soluciones de emergencia, pero de emergencia perpetua, lo cual indica que no han estimulado la equidad, ni han reducido la pobreza como prometían. Vinocur y Halperín (2004) señalan dos aspectos respecto a los programas alimentarios focalizados:

...fueron formulados para contener o solucionar efectos de las crisis sociales, en grupos específicos de la población, considerados como especialmente afectados por dichas coyunturas (...) un segundo aspecto es que se han mantenido a lo largo del tiempo, con diversas transformaciones en sus procedimientos y alcances, pero con persistencia en sus objetivos. (Vinocur y Halperín, 2004: 47)

Como afirma Ierullo (2010), esta continuidad puede explicarse a raíz de que las dinámicas y procesos generadores de la nueva marginalidad todavía no han podido ser

\footnotetext{
${ }^{6}$ El PEA es producto de una decisión política del Estado nacional de reagrupar todos los programas sociales existentes, concentrando los recursos presupuestarios en tres de ellos: emergencia alimentaria, emergencia sanitaria y emergencia ocupacional. (Para ampliar ver: Vinocur y Halperín 2004; Britos et al. 2003)

${ }^{7}$ Fuente: http://www.presidencia.gob.ar/sitios-de-gobierno/planes-de-gobierno/2661
} 
revertidos, lo cual se expresa en las condiciones de suma precariedad y marginación a la que se ven expuestos los barrios populares.

Por otro lado, la reflexión sobre las políticas del período permite identificar los rastros que los mecanismos de gestión han dejado en los cuerpos malnutridos. Considerando que los comedores funcionan como espacios en los que la comida es el eje central, sin embargo, sus prestaciones alimentarias son muy precarias.

Como sostiene Aguirre (2004), los comedores populares repiten las mismas pautas de las comidas "rendidoras" que utilizan como estrategia doméstica de consumo ${ }^{8}$ los hogares de bajos ingresos. Es decir, una comida "rendidora" es colectiva, monótona y saturada de hidratos y grasas. "El hecho de que rindan por baratos, que llenen y que gusten hace que se repitan infinitamente en las comidas cotidianas, generando dietas carentes de nutrientes necesarios y con excesos peligrosos" (Aguirre, 2004: 86).

Las estrategias de consumo están condicionadas por la oferta del mercado, y lo que permite cubrir la alimentación del hogar es la elección de alimentos rendidores. Así, "el resultado es siempre el mismo: un menú monótono que solo llena la panza del "elegido" (beneficiario)" (Boito y Huergo, 2011). Como sostienen estas autoras, los alimentos "rendidores" recuerdan siempre qué es lo posible.

Estas son las dinámicas y los procesos generadores de la nueva marginalidad que no se han revertido en treinta años de políticas alimentarias.

Retomando a Aguirre,

...para los comedores institucionales es importante seguir las pautas de alimentación del grupo porque no pueden pagar el precio del rechazo y porque este tipo de alimentación es la de menor precio. Pero el costo que se paga por no innovar y no ofrecer opciones alternativas trasciende luego a lo social. ¿Por qué? Porque el hecho de no abrir el abanico de posibilidades impide repensar la comida y sus derivaciones. Si las normas y valores representan un capital social, las instituciones asistenciales que cristalizan las representaciones populares sancionándolas como "la comida popular-institucional" ayudan, al clausurar posibilidades de elección, a empobrecer el capital cultural de la gente. (Aguirre, 2004: 85)

Los indicadores de mala alimentación también se han identificado en las prácticas de los comedores escolares. Según los estudios de CESNI,

...durante los años noventa el perfil del desenvolvimiento del PROSONU se caracterizaba por: una prestación alimentaria irregular, no sujeta a un marco

\footnotetext{
${ }^{8}$ Prácticas y representaciones acerca de la comida, realizadas por los agregados familiares, reiteradas a lo largo de su ciclo de vida, tendientes a obtener, respecto de la alimentación, una gama de satisfactores para cumplir con sus fines productivos y reproductivos. (Aguirre, 2005: 32)
} 
normativo que estableciera metas de aporte de energía y nutrientes; monotonía en el tipo de menú; una contribución nutricional cuyo perfil es similar y no complementario de las deficiencias conocidas de la alimentación hogareña; una sobrevaloración de la prestación de almuerzo en desmedro de otras de importancia estratégica como los desayunos o meriendas. (Britos et al. 2003: 19)

Estos datos indican falencias en el cumplimiento del objetivo de disminuir las condiciones deficitarias de la alimentación de los escolares.

Hacia principios del milenio los informes del CESNI afirmaban que los comedores comunitarios, si bien atienden preferencialmente a la población infantil y particularmente al grupo de niños de entre dos a cinco años que no son cubiertos por el Programa Materno Infantil ni por los comedores escolares, cubren un segmento etario más amplio, ya que concurren hermanos, madres y padres de los niños preescolares. (Britos et al. 2003: 24) Desde esta perspectiva, el desbordamiento de la demanda del comedor es congruente con la reproducción de las comidas rendidoras.

Respecto al PRANI,

...que se presentó como una oportunidad estratégica para unificar programas alimentarios y volver a ejercer alguna supervisión sobre el funcionamiento de comedores infantiles y escolares transferidos a las provincias, se convirtió rápidamente en un programa tradicional de reparto de cajas de alimentos a familias pobres, en este caso, que además fueran beneficiarias de comedores infantiles y comunitarios. (Britos et.al. 2003: 32)

En este caso, no solo es evidente la dificultad de alcanzar los objetivos propuestos, sino que es reforzado el mecanismo del reparto de cajas de alimentos, que en su mayoría, son alimentos secos, de fácil logística (respecto a los alimentos frescos), apropiados para las comidas rendidoras.

Vinocur y Halperín (2004) sostienen en cuanto a los mecanismos de control que, la dirección del programa PRANI reconoció que algunos objetivos específicos fueron variando en su operatividad debido a las diferentes realidades y necesidades provinciales. Además, se presentaron dificultades para instrumentar el diagnóstico y el monitoreo de los comedores escolares e infantiles con acuerdo de las autoridades provinciales, y su desinterés o dificultad para enfrentar reformulaciones estructurales en sus programas de asistencia alimentaria. En cuanto a la cobertura, la remisión de fondos tuvo discontinuidades, a lo que se sumó que el número de destinatarios reales superó la cantidad prevista. (Vinocur y Halperín, 2004: 29)

En el nuevo milenio, FOPAR financia la actividad de los comedores acompañados por ONG, lo cual denota un giro en el vínculo entre el Estado y los destinatarios de las políticas sociales. Como describe Massetti (2010) 
...la compleja trama de organización comunitarias que fue el sustento de la capacidad de movilización de los noventa dejó de tener un centro político único, relacionado directamente con el Estado a través del acceso a planes de microeconomía comunitaria. Lo que implicó de alguna manera la "ONGización" de las organizaciones; profesionalizando a muchos de sus cuadros. La ongización implica también una repolitización: no es ahora la protesta la forma de lograr recursos de gestión comunitaria. Sino en tal caso la cercanía o la capacidad de interlocución dentro del contexto político. (Masetti, 2010)

Para finalizar, se puede reflexionar en torno a la organización colectiva de las necesidades como una respuesta a las dificultades del Estado para garantizar una alimentación suficiente y nutricional y culturalmente adecuada, ya que persisten en su acción hasta la actualidad.

\section{Referencias Bibliográficas}

AGUIRRE, P. (2004). Ricos flacos y gordos pobres. La alimentación en crisis. Buenos Aires: Ed. Capital intelectual.

AGUIRRE, P. (2005). Estrategias de consumo: qué comen los argentinos qué comen. Buenos Aires: Mino y Dávila.

AGUIRRE, P. (2011). Reflexiones sobre las nuevas formas del hambre en el siglo XXI: la obesidad de la escasez. En: Boletín Científico Sapiens Research, Vol. 1 (2), (pp.6064).

BOITO, M-E. y HUERGO, J. (2011). El hambre como punto de origen y de llegada de las políticas alimentarias vigentes. En: Boletín Científico Sapiens Research, Vol. 1 (2), (pp. 49-53).

BRITOS, S., O'DONNELL, A., UGALDE, V. y CLACHEO, R. (2003). Programas alimentarios en Argentina. Buenos Aires: CESNI.

DE SENA, A. y CENA R. (2014). ¿Qué son las políticas sociales? Esbozos de respuestas. En De Sena, A. (ed.) Las políticas hechas cuerpo y lo social devenido emoción. (pp.1949). Ciudad Autónoma de Buenos Aires: Estudios sociológicos editora.

IÑIGO CARRERA, N.; COTARELO, M.; GOMEZ, E.; KINDGARD, F. (1995). "La revuelta Argentina 1989-1990". PIMSA. Documento de Trabajo N4

MASSETTI, A. (2010). Limitaciones de los movimientos sociales en la construcción de un estado progresista en Argentina. Argumentos. Revista de crítica social. Buenos Aires, Octubre (pp. 82-108).

MASSETTI, A. (2011). Las tres transformaciones de la política pública asistencial y su relación con las organizaciones sociopolíticas (2003-2009). Entramados y perspectivas. Revista de la carrera de Sociología. Buenos Aires, Junio. (pp. 9-36) 
VINOCUR, P. y HALPERÍN, L. (2004). Pobreza y políticas sociales en Argentina de los años noventa. Santiago de Chile: CEPAL. Naciones Unidas.

\section{Referencias electrónicas}

IERULLO, M. (2010). Organizaciones comunitarias y atención de las necesidades básicas. Los comedores comunitarios frente a los procesos de pauperización en el Área Metropolitana de Buenos Aires (AMBA). Revista Margen. Edición N059. Primavera 2010. Buenos Aires.

Disponible en: http://www.margen.org/suscri/margen59/ieru.pdf Fecha de acceso: 24 de junio de 2014.

SCRIBANO, A.; EYNARD, M. y HUERGO, J. (2010). Alimentación energía y depredación de los bienes comunes: la invisibilidad de la expropiación colonial. En Onteaiken. Boletín sobre Prácticas y Estudios de Acción Colectiva. № 9, Año 5.

Disponible en: www.accioncolectiva.com.ar

Fecha de acceso: 6 de abril de 2014. 